\title{
Grassmann techniques applied to classical spin systems
}

\author{
M. Clusel ${ }^{1}$, J.-Y. Fortin ${ }^{2}$ \\ 1 Department of Physics and Center for Soft Matter Research, New-York University, 4 Washington place, \\ New-York NY 10003, USA \\ 2 Institut Jean Lamour Département de Physique de la Matière et des Matériaux, Groupe de Physique \\ Statistique CNRS - Nancy-Université BP 70239 F-54506 Vandoeuvre les Nancy Cedex, France
}

Received April 22, 2009, in final form May 20, 2009

\begin{abstract}
We review problems involving the use of Grassmann techniques in the field of classical spin systems in two dimensions. These techniques are useful to perform exact correspondences between classical spin Hamiltonians and field-theory fermionic actions. This contributes to a better understanding of critical behavior of these models in term of non-quadratic effective actions which can be seen as an extension of the free fermion Ising model. Within this method, identification of bare masses allows for an accurate estimation of critical points or lines and which is supported by Monte-Carlo results and diagrammatic techniques.
\end{abstract}

Key words: Grassmann algebra, spin systems, critical phenomena

PACS: $02.30 .1 k, 05.50 .+q, 05.70 . F h$

\section{Introduction}

Classical and quantum spin models such as Ising model play an important role in the field of statistical physics as they allow for an accurate understanding of critical phenomena in general. Many techniques $[1,2]$ were developed in order to deal with the difficulty of estimating the partition function and other thermodynamical properties in the critical region in dimension more than one. An exact mathematical description of the two-dimensional (2D) Ising model relies on the JordanWigner transformation [3] which maps the product of Boltzmann weights onto a fermionic action of free fermions with a mass vanishing at the second order critical temperature given in dimensionless units $T_{\mathrm{c}}=2 / \ln (1+\sqrt{2}) \simeq 2.2691851$. Also a method based on the correspondence between the Ising model and dimer problems [4] uses the notion of Pfaffians, which are directly connected to integrals over Grassmannian objects. Both fermions and Grassmann variables are therefore closely tied to the Ising model. A direct introduction of Grassmann variables as an alternative tool to solve the Ising model was done long ago in the 80's by Bugrij [5] and Plechko [6] (see also a later discussion by Nojima [7]). It is based on a simple integral representation of the individual Boltzmann weights and which has the property to decouple the spins. The price to pay is a noncommutativity of terms arising from this representation. In order to deal with this particular representation, Bugrij used two families of Grassmann variables which commute with each other, then identified the resulting functional integral of the partition function with a determinant. From another point of view, Plechko introduced symmetries which order the non-commuting quantities so that the sum over the spins can be performed exactly. In this paper we review the process of how to generalize Plechko's method for Blume-Capel model [8], which is the simplest model beyond Ising, to spin-S Ising models and how to construct an exact fermionic action for each case. This would provide a natural extension of the exact fermionic quadratic action found for the spin $S=1 / 2$ Ising model. In particular, we will build on previous work on the Blume-Capel (BC) case $S=1$ [8] where a line of second-order critical points is terminated by a tricritical point. This is the next case beyond the Ising model and which possesses a rich critical behavior. This model was used to qualitatively explain the phase transition in a mixture of $\mathrm{He}^{3}-\mathrm{He}^{4}$ adsorbed on a $2 \mathrm{D}$ 
surface [9]. Below a concentration of $67 \%$ in $\mathrm{He}^{3}$, the mixture undergoes a $\lambda$ transition and the two components separate through a first order phase transition with only $\mathrm{He}^{4}$ superfluid. On a 2D lattice, He atoms are represented by a spin-like variable, according to the following rule: an $\mathrm{He}^{3}$ atom is associated to the value 0 , whereas a $\mathrm{He}^{4}$ is represented by a classical Ising spin taking the values \pm 1 . Within this framework, all the lattice sites are occupied either by an $\mathrm{He}^{3} \mathrm{or} \mathrm{He}^{4}$ atom. In addition to nearest-neighbor interactions, the energy includes a term $\Delta_{0} \sum_{m n} S_{m n}^{2}$, with $S_{m n}^{2}=0,1$, to take into account a possible change in vacancies number. $\Delta_{0}$ can be viewed as a chemical potential for vacancies, or as a parameter of crystal field in a magnetic interpretation of atomic physics. It would be of particular interest to have a fermionic description of the BC model in order to obtain more information about the kind of interaction fermions living on the 2D lattice have compared with the Ising free fermion case.

So, one of the main questions is how to obtain a generic fermionic action for a spin-S model and what this method teaches us for the $\mathrm{BC}$ model in particular. We explain in the next section the main ideas of this method.

\section{Description of the fermionization for general classical spin-S models}

Let us consider the following Hamiltonian on a 2D lattice of size $L \times L$

$$
H=-\sum_{m=1}^{L} \sum_{n=1}^{L} J\left[S_{m n} S_{m+1 n}+S_{m n} S_{m n+1}\right]+\Delta_{0} \sum_{m=1}^{L} \sum_{n=1}^{L} S_{m n}^{2},
$$

where $J$ is the Ising coupling constant and $\Delta_{0}$ is the splitting crystal field or represents a chemical potential in the $\mathrm{BC}$ case. In particular for $\Delta_{0}$ large and positive, it favors small spin values. This crystal field can be replaced by any potential $V\left(S_{m n}^{2}\right)$ depending on the square of the local spin. Spins $S_{m n}$ take $2 S+1$ values with $S_{m n}=-S,-S+1, \cdots, S$. The partition function is the sum over all possible spin configurations $\mathcal{Z}=\operatorname{Tr} \exp (-\beta H)$. $\mathcal{Z}$ contains products of the Boltzmann weights $\exp \left(K S_{m n} S_{m+1 n}\right)$ (where $S_{m n}$ and $S_{m+1 n}$ are neighboring spins and $K=J / k_{\mathrm{B}} T$ ) which take $q+1=S(S+1)+1$ different values if $S$ is an integer, and $q+1=(S+1 / 2)(S+3 / 2)$ values if $S$ is half-integer. Since there are $q+1$ possible values for each Boltzmann weight, we can project each of them onto a polynomial function of degree $q$ in the variable $S_{m n} S_{m+1 n}$ :

$$
\exp \left(K S_{m n} S_{m+1 n}\right)=\sum_{k=0}^{q} u_{k}\left(S_{m n} S_{m+1 n}\right)^{k}=u_{0} \prod_{\alpha=1}^{q}\left(1+x_{\alpha} S_{m n} S_{m+1 n}\right)
$$

where the $q+1$ constants $u_{k}$ are determined by solving the linear system of $q+1$ equations satisfied by the above relation. To see on specific examples how it works, let us consider first the Ising case, $S=1 / 2$. Since $S$ is half integer, we have $q=1$. Therefore,

$\exp \left(K S_{m n} S_{m+1 n}\right)=\operatorname{ch}(K / 4)+4 \operatorname{sh}(K / 4) S_{m n} S_{m+1 n}, \quad u_{0}=\operatorname{ch}(K / 4), \quad u_{1}=4 \operatorname{sh}(K / 4)$.

In the Blume-Capel model, since $S$ is integer, we have $q=2$ and it is straightforward to show that

$$
\begin{aligned}
\exp \left(K S_{m n} S_{m+1 n}\right) & =1+\operatorname{sh}(K) S_{m n} S_{m+1 n}+(\operatorname{ch}(K)-1)\left(S_{m n} S_{m+1 n}\right)^{2} \\
u_{0} & =1, \quad u_{1}=\operatorname{sh}(K), \quad u_{2}=\operatorname{ch}(K)-1
\end{aligned}
$$

For $S$ integer the first coefficient $u_{0}$ is always unity, and from equation (2) we can write

$$
u_{k}=u_{0} \sum_{\alpha_{1}<\alpha_{2}<\cdots<\alpha_{k}} x_{\alpha_{1}} x_{\alpha_{2}} \cdots x_{\alpha_{k}}, \quad 1 \leqslant k \leqslant q .
$$

We will set for convenience in the following $u_{k \geqslant q+1}=0$ since the polynoms are all finite. Our purpose is to transform the partition function $\mathcal{Z}$ which is a sum over spin variables into a multiple integral over Grassmann variables. For this, let us introduce $q$ pairs of Grassmann variables $[6,8,10]$ 
$\left(a_{m n}^{\alpha}, \bar{a}_{m n}^{\alpha}\right)$ on each site for the horizontal direction and $q$ additional pairs $\left(b_{m n}^{\alpha}, \bar{b}_{m n}^{\alpha}\right)$ for the vertical direction. Here $\alpha$ takes the values $1, \ldots, q$. There are, therefore, $4 q$ Grassmann variables at each site of the lattice. In particular, the Ising model is represented by two pairs of Grassmann variables per site which can afterward be reduced to only one pair [6]. For each couple of terms

$$
\left(1+x_{\alpha} S_{m n} S_{m+1 n}\right)\left(1+x_{\alpha} S_{m n} S_{m n+1}\right)
$$

appearing in the partition function, we introduce the following integral representation

$$
\begin{array}{r}
1+x_{\alpha} S_{m n} S_{m+1 n}=\int \mathrm{d} \bar{a}_{m n}^{\alpha} \mathrm{d} a_{m n}^{\alpha} \mathrm{e}^{a_{m n}^{\alpha} \bar{a}_{m n}^{\alpha}}\left(1+a_{m n}^{\alpha} S_{m n}\right)\left(1+x_{\alpha} \bar{a}_{m n}^{\alpha} S_{m+1 n}\right), \\
1+x_{\alpha} S_{m n} S_{m n+1}=\int \mathrm{d} \bar{b}_{m n}^{\alpha} \mathrm{d} b_{m n}^{\alpha} \mathrm{e}^{b_{m n}^{\alpha} \bar{b}_{m n}^{\alpha}}\left(1+b_{m n}^{\alpha} S_{m n}\right)\left(1+x_{\alpha} \bar{b}_{m n}^{\alpha} S_{m n+1}\right) .
\end{array}
$$

From the last expression, we introduce the link factors $A_{m n}^{\alpha}=1+a_{m n}^{\alpha} S_{m n}, \bar{A}_{m+1 n}^{\alpha}=1+$ $x_{\alpha} \bar{a}_{m n}^{\alpha} S_{m+1 n}, B_{m n}^{\alpha}=1+b_{m n}^{\alpha} S_{m n}$, and $\bar{B}_{m n+1}^{\alpha}=1+x_{\alpha} \bar{b}_{m n}^{\alpha} S_{m n+1}$, so that the partition function can be written as

$$
\mathcal{Z}=u_{0}^{2 L^{2}} \operatorname{Tr}_{\{S, a, b\}}\left[\prod_{m n} \mathrm{e}^{\Delta S_{m n}^{2}} \times\left[\prod_{\alpha}\left(A_{m n}^{\alpha} \bar{A}_{m+1 n}^{\alpha}\right) \prod_{\beta}\left(B_{m n}^{\beta} \bar{B}_{m n+1}^{\beta}\right)\right]\right],
$$

where $\Delta=-\beta \Delta_{0}$. The mixed trace operator introduced in the last expression is defined by the following sums and integrals:

$$
\operatorname{Tr}_{\{S, a, b\}}[.]=\operatorname{Tr}_{\{S\}} \int\left[\prod_{m n, \alpha} \mathrm{d} \bar{a}_{m n}^{\alpha} \mathrm{d} a_{m n}^{\alpha} \mathrm{d} \bar{b}_{m n}^{\alpha} \mathrm{d} b_{m n}^{\alpha} \times \mathrm{e}^{a_{m n}^{\alpha} \bar{a}_{m n}^{\alpha}+b_{m n}^{\alpha} \bar{b}_{m n}^{\alpha}}\right][.] .
$$

Inside the integral symbols, the pairs of Grassmannian link factors in brackets

$$
\left(A_{m n}^{\alpha} \bar{A}_{m+1 n}^{\alpha}\right), \quad\left(B_{m n}^{\alpha} \bar{B}_{m n+1}^{\alpha}\right)
$$

can be moved freely with the other terms since they correspond to commutative scalars after integration. In particular, it is convenient to rearrange the products over $\alpha$ in order to put together the link factors of different $\alpha$ with the same site indices $(m, n)$ using the mirror ordering symmetry introduced in Plechko's method [6] in the context of the 2D Ising model, and which is still relevant in the spin-S case:

$$
\begin{aligned}
\prod_{\alpha=1}^{q}\left(A_{m n}^{\alpha} \bar{A}_{m+1 n}^{\alpha}\right) & =\left(A_{m n}^{1} \bar{A}_{m+1 n}^{1}\right) \ldots\left(A_{m n}^{q} \bar{A}_{m+1 n}^{q}\right) \\
& =\left(A_{m n}^{1}\left(A_{m n}^{2} \ldots\left(A_{m n}^{q-1}\left(A_{m n}^{q} \bar{A}_{m+1 n}^{q}\right) \bar{A}_{m+1 n}^{q-1}\right) . . \bar{A}_{m+1 n}^{1}\right)\right. \\
& =\left(\overline{\prod_{\alpha=1}^{q}} A_{m n}^{\alpha}\right)\left(\overleftarrow{\prod_{\alpha=1}^{q}} \bar{A}_{m+1 n}^{\alpha}\right)
\end{aligned}
$$

where the arrows indicate that the product is ordered, i. e. increasing label $\alpha$ in the first product from left to the right and in the second one from right to the left. For convenience, we will use the notation

$$
\mathcal{O}_{m n}=\prod_{\alpha} A_{m n}^{\alpha}, \quad \overline{\mathcal{O}}_{m+1 n}=\prod_{\alpha} \bar{A}_{m+1 n}^{\alpha}
$$

for objects on the horizontal links and

$$
\mathcal{P}_{m n}=\prod_{\alpha} B_{m n}^{\alpha}, \quad \overline{\mathcal{P}}_{m n+1}=\prod_{\alpha} \bar{B}_{m n+1}^{\alpha}
$$


for the ones on vertical links. Then the partition function can be rewritten as

$$
\begin{aligned}
\mathcal{Z}= & u_{0}^{2 L^{2}} \operatorname{Tr}_{\{S\}} \int\left[\prod_{m n, \alpha} \mathrm{d} \bar{a}_{m n}^{\alpha} \mathrm{d} a_{m n}^{\alpha} \mathrm{d} \bar{b}_{m n}^{\alpha} \mathrm{d} b_{m n}^{\alpha} \mathrm{e}^{a_{m n}^{\alpha} \bar{a}_{m n}^{\alpha}+b_{m n}^{\alpha} \bar{b}_{m n}^{\alpha}}\right] \\
& \times \prod_{m n} \mathrm{e}^{\Delta S_{m n}^{2}}\left(\mathcal{O}_{m n} \overline{\mathcal{O}}_{m+1 n}\right)\left(\mathcal{P}_{m n} \overline{\mathcal{P}}_{m n+1}\right) \\
\equiv & u_{0}^{2 L^{2}} \operatorname{Tr}_{\{S, a, b\}}\left[\prod_{m n} \mathrm{e}^{\Delta S_{m n}^{2}}\left(\mathcal{O}_{m n} \overline{\mathcal{O}}_{m+1 n}\right)\left(\mathcal{P}_{m n} \overline{\mathcal{P}}_{m n+1}\right)\right]
\end{aligned}
$$

At this stage of the algebra, we use the mirror and associative symmetries which were used for solving the Ising model $[6,10]$ and which are still valid here to rearrange the operators $\mathcal{O}$ and $\mathcal{P}$. In principle boundary terms should be treated separately in order to obtain the exact finite size partition function depending on boundary conditions [6] but they are not relevant in the thermodynamical limit $L \rightarrow \infty$ we are interested in here. Instead, here we consider the simple case of free boundary conditions, and we obtain the exact expression after rearrangement of the $\mathcal{O}$ and $\mathcal{P}$ operators:

$$
\mathcal{Z}=u_{0}^{2 L^{2}} \operatorname{Tr}_{\{S, a, b\}}\left[\overrightarrow{\prod_{n=1}^{L}}\left(\prod_{m=1}^{L} \mathrm{e}^{\Delta S_{m n}^{2}}\left(\overline{\mathcal{O}}_{m n} \overline{\mathcal{P}}_{m n} \mathcal{O}_{m n}\right) \overleftrightarrow{\prod_{m=1}^{L}} \mathcal{P}_{m n}\right)\right]
$$

Now, from this expression, the spins can individually be summed up from $S_{L n}$ to $S_{1 n}$ for any given $n$. We will need to introduce the following weights $W_{m n}$ which include all the dependence on the individual spins $S_{m n}$

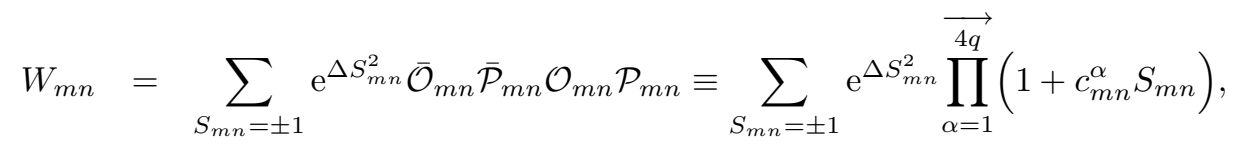

where we have defined the following $4 q$ sets of Grassmann variables $c_{m n}^{\alpha}$ in the following order:

$$
\begin{aligned}
c_{m n}^{1} & =x_{q} \bar{a}_{m-1 n}^{q}, c_{m n}^{2}=x_{q-1} \bar{a}_{m-1 n}^{q-1}, \cdots, c_{m n}^{q}=x_{1} \bar{a}_{m-1 n}^{1}, \\
c_{m n}^{q+1} & =x_{q} \bar{b}_{m n-1}^{q}, c_{m n}^{q+2}=x_{q-1} \bar{b}_{m n-1}^{q-1}, \cdots, c_{m n}^{2 q}=x_{1} \bar{b}_{m n-1}^{1} \\
c_{m n}^{2 q+1} & =a_{m n}^{1}, c_{m n}^{2 q+2}=a_{m n}^{2}, \cdots, c_{m n}^{3 q}=a_{m n}^{q} \\
c_{m n}^{3 q+1} & =b_{m n}^{1}, c_{m n}^{3 q+2}=b_{m n}^{2}, \cdots, c_{m n}^{4 q}=b_{m n}^{q} .
\end{aligned}
$$

The sum over $S_{m n}= \pm 1$ in equation (12) can be performed by noticing that only products involving an even number of $S_{m n}$ give a non-zero contribution. We also define the scalars (we remind that $\left.\Delta=-\beta \Delta_{0}\right)$

$$
\alpha_{k}=\sum_{S_{m n}=-S}^{S} S_{m n}^{2 k} \exp \left(\Delta S_{m n}^{2}\right)
$$

and the ordered products

$$
q_{m n}^{(k)}=\sum_{\alpha_{1}<\alpha_{2}<\cdots<\alpha_{k}} c_{m n}^{\alpha_{1}} c_{m n}^{\alpha_{2}} \cdots c_{m n}^{\alpha_{k}}, \quad q_{m n}^{(0)} \equiv 1
$$

with $q_{m n}^{(4 q)}=c_{m n}^{1} \cdots c_{m n}^{4 q}$ the term of highest degree in Grassmann variables. Using these quantities, it is easy to show that the partial Boltzmann weights (12) are given by

$$
W_{m n}=\sum_{k=0}^{2 q} \alpha_{k} q_{m n}^{(2 k)}
$$


Then the fermionic representation of the partition function can be expressed as a multiple integral over Grassmannian variables only

$$
\mathcal{Z}=u_{0}^{2 L^{2}} \operatorname{Tr}_{\{a, b, \bar{a}, \bar{b}\}} \prod_{m n} W_{m n}
$$

For small values of $S$, the weights $W_{m n}$ can be exponentiated so that a fermionic action can be defined. Indeed, since the first term of $W_{m n}$ is the pure scalar $\alpha_{0}$ and the others are products of pure commutating Grassmannian objects, it is tempting to exponentiate the sum (16) to obtain directly a fermionic action. This comes from the simple observation that for any Grassmann variable $a$, we have $1+a=\mathrm{e}^{a}$. Of course, the exponentiation of the sum (16) is more complicated. For example, for commuting objets $a$ and $b$ such as the $q_{m n}^{k}$ s, we have $1+a+b=\exp (1+a+b-a b)$. In this case the order of the polynomial object inside the exponential is bigger than in the original sum since the extra counter-term $a b$ is necessary for the identity to be exact. Moreover, these weights are connected by nearest-neighbor interactions hidden in the variables $c_{m n}^{\alpha}$. In the case of the Ising model, where the exponentiation can be done quite easily, the argument of the exponential is purely quadratic in the $c_{m n}^{\alpha}$ 's and, therefore, the partition function can be integrated out with the use of a determinant or a Bogoliubov transformation in the Fourier space. Moreover, the $4 q=4$ Grassmann variables in this case can be reduced to 2 by partial integration of non-relevant variables. In the $\mathrm{BC}$ model, the argument is a polynomial of degree 8 in Grassmann variables since there are 8 independent variables $(4 q=8)$. In general we naturally expect the argument to be at most a polynomial of degree $4 q$ in these variables, which can be reduced or not by partial integrations. Except for the case $q=1$, however, the partition function cannot be expressed as a determinant, so that a full exact solution of the partition function cannot be found this way. If the action is quadratic, the use of the following Gaussian integral [11], defined on Grassmann set of variables $\left\{a_{i}, \bar{a}_{i}\right\}_{i=1, \ldots, N}$, and for a square matrix $A$

$$
\int \prod_{i=1}^{N} \mathrm{~d} \bar{a}_{i} \mathrm{~d} a_{i} \exp \left(\sum_{i, j=1}^{N} a_{i} A_{i j} \bar{a}_{j}\right)=\operatorname{det} A
$$

allows us to express the partition function as a determinant. Quadratic fermionic form in the exponential (18) is typically called action for a free-field theory. When the action is non-quadratic, the integral is not Gaussian and cannot be expressed as a determinant, which yields in principle to a non-integrable theory. However, physical information such as bare masses (see last section) can be extracted from these non-quadratic actions which represent generic theories of interacting fermions.

\section{Fermionic action of the Blume-Capel model}

In this section, we consider the case $S=1$ (Blume-Capel model) which is the simplest example of a classical spin beyond the Ising model. It possesses in the phase diagram $\left(T, \Delta_{0}\right)$ a second-order critical line separating an ordered phase from a disordered one and terminated by a tricritical point (see figure 1). From the previous section equation (17) allows us to write an action after exponentiation of the Grassmann variables which can be done exactly after some tedious algebra. The 4 pairs of variables per site can, however, be reduced to 2 pairs by partial integration. Another simpler way of obtaining this BC fermionic action is possible [12] using the $Z_{2}$ symmetry of the spin variables $S_{m n}$. Indeed the partition function is invariant if we perform the gauge transformation $S_{m n} \rightarrow \sigma_{m n} S_{m n}$ with $\sigma_{m n}= \pm 1$. In this case it is possible to simplify the process of the previous method and write an action containing only 2 pairs of variables per site instead of 4 :

$$
\begin{aligned}
\mathcal{Z}= & \left(2 \mathrm{e}^{\Delta} \cosh ^{2} K\right)^{L^{2}} \int \prod_{m=1}^{L} \prod_{n=1}^{L} \mathrm{~d} \bar{a}_{m n} \mathrm{~d} a_{m n} \mathrm{~d} \bar{b}_{m n} \mathrm{~d} b_{m n} \exp \left\{\sum_{m=1}^{L} \sum_{n=1}^{L}\right. \\
& {\left[a_{m n} \bar{a}_{m n}+b_{m n} \bar{b}_{m n}+a_{m n} b_{m n}+t\left(\bar{a}_{m-1 n}+\bar{b}_{m n-1}\right)\left(a_{m n}+b_{m n}\right)+t^{2} \bar{a}_{m-1 n} \bar{b}_{m n-1}\right.}
\end{aligned}
$$




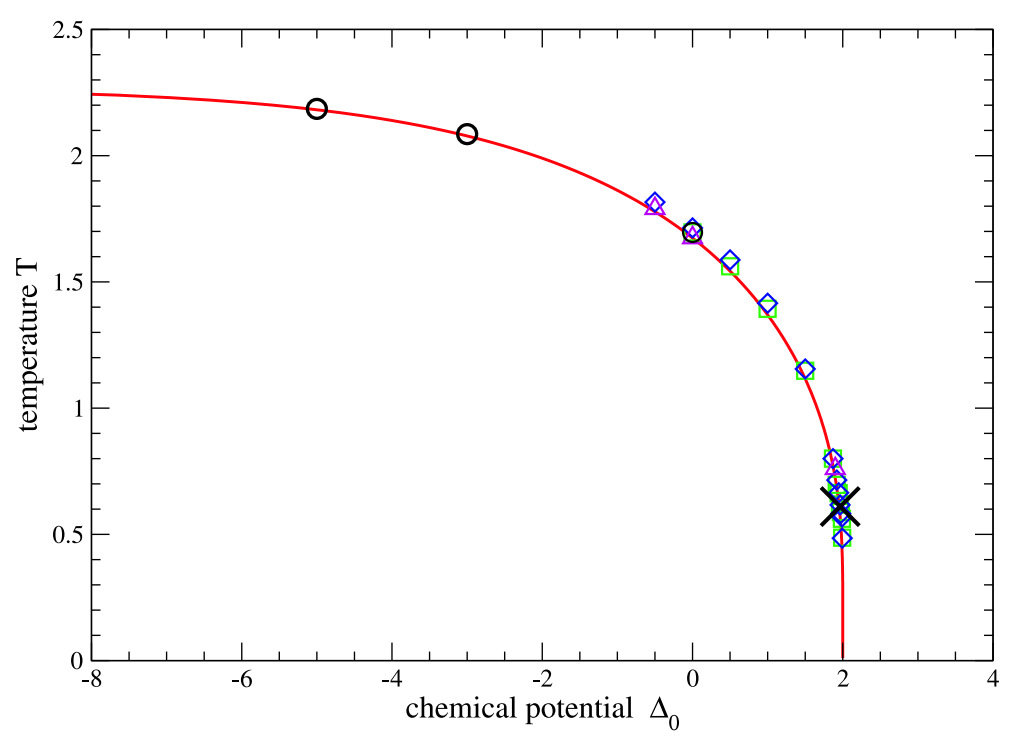

Figure 1. (color online) Figure taken from reference [12] showing comparison between critical line defined by the vanishing mass (35) (plane red line) and numerical results from Monte Carlo simulations. The black dots are from figure 1, da Silva et al. [19] (Wang-Landau method). The cross symbol indicates the tricritical point identified by [12] using a Hartree-Fock-Bogoliubov approximation for the quartic part (33) of the effective action. The blue diamond symbols are from [20], the magenta triangles from [21], and the green squares from [22] (see also table 1 for other numerical values at $\left.\Delta_{0}=0\right)$.

$$
\left.\left.+\quad g_{0} a_{m n} \bar{a}_{m n} b_{m n} \bar{b}_{m n} \exp \left(-\gamma a_{m-1 n} \bar{a}_{m-1 n}-\gamma b_{m n-1} \bar{b}_{m n-1}-t^{2} \bar{a}_{m-1 n} \bar{b}_{m n-1}\right)\right]\right\}
$$

where we have introduced the following constants:

$$
g_{0}=\frac{\mathrm{e}^{-\Delta}}{2 \cosh ^{2} K}, \quad \gamma=1-\frac{1}{\cosh K}=1-\sqrt{1-t^{2}}, \quad t=\tanh K
$$

The fermionic integral (19) is the exact expression even for a finite lattice, provided we assume free boundary conditions for both spins and fermions. The other possible form for the partition function with periodic boundary conditions in both directions can be written in a similar way as the Ising model on a torus $[6,13,14]$. The partition function would be the sum of 4 fermionic integrals with periodic-antiperiodic boundary conditions for the fermions. In the expression (19), we can recognize the sum of the Ising action, which here appears as the Gaussian part of the total action $[6,10]$ :

$S_{\text {Ising }}=\sum_{m, n=1}^{L} a_{m n} \bar{a}_{m n}+b_{m n} \bar{b}_{m n}+a_{m n} b_{m n}+t\left(\bar{a}_{m-1 n}+\bar{b}_{m n-1}\right)\left(a_{m n}+b_{m n}\right)+t^{2} \bar{a}_{m-1 n} \bar{b}_{m n-1}$,

and a non-quadratic interaction part, which is a polynomial of degree 8 in Grassmann variables (which can be seen if we expand the exponential inside the action):

$$
S_{\mathrm{int}}=g_{0} \sum_{m, n=1}^{L} a_{m n} \bar{a}_{m n} b_{m n} \bar{b}_{m n} \exp \left(-\gamma a_{m-1 n} \bar{a}_{m-1 n}-\gamma b_{m n-1} \bar{b}_{m n-1}-t^{2} \bar{a}_{m-1 n} \bar{b}_{m n-1}\right)
$$

This allows us to rewrite the partition function as a fermionic field-theory in a compact form

$$
\mathcal{Z}=\left(2 \mathrm{e}^{\Delta} \cosh ^{2} K\right)^{L^{2}} \int \mathcal{D} \bar{a} \mathcal{D} a \mathcal{D} \bar{b} \mathcal{D} b \exp \left(S_{\text {Ising }}+S_{\text {int }}\right)
$$


The BC model differs from the Ising model by the interaction term in the action (21) which is not quadratic. Therefore, the BC model is not solvable in the sense of free fermions as a determinant of some matrix, unlike the $2 \mathrm{D}$ Ising model.

\subsection{Mixed representation of the $\mathrm{BC}$ model}

The coupling of Grassmann variables in equation (21) prevents us from integrating further and reducing the number of variables per site unlike the Ising model where the minimal action contains one pair only $[7,18]$. The minimal action of the Ising model admits an interpretation in term of Dirac representation of free fermions which become massless at the critical point. In the previous work we were able to reduce the number of Grassmann variables by partially introducing hard core bosons in the previous action, since terms such as $\eta_{m n}=a_{m n} \bar{a}_{m n}$ or $\tau_{m n}=b_{m n} \bar{b}_{m n}$ may have an interpretation of local densities or occupation numbers. Variables $\eta_{m n}$ and $\tau_{m n}$ are commuting and nilpotent, $\eta_{m n}^{2}=\tau_{m n}^{2}=0$. We can replace the quantities depending on $a_{m n} \bar{a}_{m n}$ and $b_{m n} \bar{b}_{m n}$, especially in the interaction part, by their respective nilpotent variables, using, for this task, a general definition of Dirac distribution for any polynomial function $f$ of $a_{m n} \bar{a}_{m n}$ or $b_{m n} \bar{b}_{m n}$ [12]:

$$
\begin{aligned}
f\left(a_{m n} \bar{a}_{m n}\right) & =\int \mathrm{d} \eta_{m n} \mathrm{~d} \bar{\eta}_{m n} f\left(\eta_{m n}\right) \exp \left[\bar{\eta}_{m n}\left(\eta_{m n}+a_{m n} \bar{a}_{m n}\right)\right], \\
f\left(b_{m n} \bar{b}_{m n}\right) & =\int \mathrm{d} \tau_{m n} \mathrm{~d} \bar{\tau}_{m n} f\left(\tau_{m n}\right) \exp \left[\bar{\tau}_{m n}\left(\tau_{m n}+b_{m n} \bar{b}_{m n}\right)\right] .
\end{aligned}
$$

A natural definition [15] of the integrals involving commuting nilpotent variables is to impose the following rules (and similar for $\bar{\eta}_{m n}, \bar{\tau}_{m n}$ ):

$$
\int \mathrm{d} \eta_{m n}\left(1, \eta_{m n}\right)=(0,1), \quad \int \mathrm{d} \tau_{m n}\left(1, \tau_{m n}\right)=(0,1)
$$

This change of variables allows us to integrate over the $a_{m n}$ 's and $b_{m n}$ 's in the new action. One advantage is that after this operation there are only two fermionic variables per site, although two additional pairs of bosonic variables have been introduced. In fact we can integrate over one pair of bosonic variables [12], for example $\bar{\eta}_{m n}, \bar{\tau}_{m n}$, using the help of integration rules and Dirac function given by (23). At the end, it remains a mixed action made of one pair per site of fermionic and bosonic variables respectively, with an interaction between fermions and bosons. A convenient replacement of the variables $\bar{a}_{m n}$ by $c_{m n}$ and $\bar{b}_{m n}$ by $-\bar{c}_{m n}$ in the final integral leads us to isolate the minimal local action for the pure Ising model $[16,17]$ with one pair of Grassmann variables per site:

$$
S_{\text {Ising }}=c_{m n} \bar{c}_{m n}+t\left(c_{m n}+\bar{c}_{m n}\right)\left(c_{m-1 n}-\bar{c}_{m n-1}\right)-t^{2} c_{m-1 n} \bar{c}_{m n-1}
$$

and the interaction part

$$
S_{\mathrm{int}}=g_{0} \sum_{m, n} \eta_{m n} \tau_{m n}\left[\left(1-\gamma \eta_{m-1 n}\right)\left(1-\gamma \tau_{m n-1}\right)+t^{2} c_{m-1 n} \bar{c}_{m n-1}\right]
$$

with the quantities

$$
\begin{aligned}
& \bar{q}_{m n}=c_{m n} \bar{c}_{m n}+t c_{m n}\left(c_{m-1 n}-\bar{c}_{m n-1}\right)=c_{m n}\left[\bar{c}_{m n}+t\left(c_{m-1 n}-\bar{c}_{m n-1}\right)\right], \\
& q_{m n}=c_{m n} \bar{c}_{m n}+t \bar{c}_{m n}\left(c_{m-1 n}-\bar{c}_{m n-1}\right)=\left[c_{m n}-t\left(c_{m-1 n}-\bar{c}_{m n-1}\right)\right] \bar{c}_{m n} .
\end{aligned}
$$

The Ising part is the same action that results from the integration over $a_{m n}, b_{m n}$ from the original Ising case. The introduction of nilpotent variables was necessary to achieve this partial extraction of the Ising contribution. The physical interpretation of the previous mixed representation is that it can be possible to describe the BC model with fermionic variables for the states $S= \pm 1$ and bosonic ones for states $S=0$. In the limit $\Delta_{0} \rightarrow-\infty$, the system is completely described in terms of fermions (Ising sector), while when $\Delta_{0}$ is increasing, fermions and bosons begin to interact. Beyond a critical value of $\Delta_{0}$, fermions form bosonic pairs and in the limit $\Delta_{0} \rightarrow+\infty$, all fermions condense into bosons, leading to a purely bosonic system. This view should be supported by further analysis. 


\subsection{Corrections to the effective action in the continuum limit}

The integration of the previous action (26) over variables $\left(\eta_{m n}, \tau_{m n}\right)$ can be performed perturbatively, as part of an expansion in the low momentum limit. We will formally define the derivatives of Grassmann variables [18], $\partial_{x} c_{m n}=c_{m n}-c_{m-1 n}$ and $\partial_{y} c_{m n}=c_{m n}-c_{m n-1}$ in the limit of large $L$. In this limit and in the Fourier space, the high order derivatives account in the action for a small contribution in momenta $\mathbf{k}=2 \pi(m, n) / L$, with $m, n \ll L$ positive integers. We would like to obtain in this limit the non-trivial part of the non-quadratic interaction in terms of variables $c_{m n}, \bar{c}_{m n}$ only. The procedure is described in reference [12] and based partially on substitution rules such as

$$
\eta_{m n} \tau_{m n} \rightarrow c_{m n} \bar{c}_{m n}, \quad \eta_{m n} \rightarrow \bar{q}_{m n}, \quad \tau_{m n} \rightarrow q_{m n}
$$

There are unfortunately more complicated terms in the resulting action than by using the substitution rules alone, such as

$$
g_{0}^{2} \gamma^{2} c_{m n} \bar{c}_{m n} c_{m+1 n} \bar{c}_{m+1 n} c_{m n+1} \bar{c}_{m n+1},
$$

but they can be discarded in the approximation scheme above in the sense they correspond to corrective terms higher than quartic polynomials or quantities of the order of $\mathcal{O}\left(g_{0}\right)$ where $g_{0}$ is the natural parameter of the expansion. It is exponentially small in the region where $\Delta_{0}$ is large and negative (Ising behavior). At the lowest order we found that the effective action (25), (26) can be approximated by the following expansion with respect to $g_{0}$

$$
\begin{aligned}
\mathcal{S}_{\text {effective }}= & S_{\text {Ising }}+g_{0} \sum_{m, n} c_{m n} \bar{c}_{m n}\left[\left(1-\gamma \bar{q}_{m-1 n}\right)\left(1-\gamma q_{m n-1}\right)+t^{2} c_{m-1 n} \bar{c}_{m n-1}\right] \\
& +g_{0}^{2} \gamma^{2} \sum_{m, n} c_{m n} \bar{c}_{m n} c_{m+1 n} \bar{c}_{m+1 n} c_{m n+1} \bar{c}_{m n+1}+\cdots
\end{aligned}
$$

From the previous result, it appears to be suitable to express the quadratic and quartic parts in the Fourier space (in the large but finite $L$ limit), where we define the following transformations

$$
c(\mathbf{r})=\frac{1}{L} \sum_{\mathbf{k}} c_{\mathbf{k}} \exp (\mathrm{ik} \cdot \mathbf{r}), \quad \bar{c}(\mathbf{r})=\frac{1}{L} \sum_{\mathbf{k}} \bar{c}_{\mathbf{k}} \exp (-\mathrm{ik} \cdot \mathbf{r}) .
$$

The Ising part of the action can be written as

$$
S_{\text {Ising }}=\sum_{\mathbf{k} \in S}\left[m_{\mathrm{BC}}+\mathrm{i} t(t+1)\left(k_{x}-k_{y}\right)\right]\left(c_{\mathbf{k}} \bar{c}_{\mathbf{k}}-c_{-\mathbf{k}} \bar{c}_{-\mathbf{k}}\right)+2 \mathrm{i} t k_{x} c_{\mathbf{k}} c_{-\mathbf{k}}+2 \mathrm{i} t k_{y} \bar{c}_{\mathbf{k}} \bar{c}_{-\mathbf{k}}
$$

with $m_{\mathrm{BC}}=1-2 t-t^{2}+g_{0}$ and the quartic term can be expressed as

$$
S_{\mathrm{int}}=g_{0} \frac{1}{L^{2}} \sum_{\mathbf{k}^{\prime}, \mathbf{k}^{\prime \prime}, \mathbf{q}} V_{\mathbf{k}^{\prime \prime}, \mathbf{k}^{\prime \prime}-\mathbf{q}} c_{\mathbf{k}^{\prime}} c_{\mathbf{k}^{\prime \prime}} \bar{c}_{\mathbf{k}^{\prime}+\mathbf{q}} \bar{c}_{\mathbf{k}^{\prime \prime}-\mathbf{q}},
$$

with the potential

$$
\begin{gathered}
V_{\mathbf{k}, \mathbf{k}^{\prime}}=-\alpha k_{x} k_{y}^{\prime}+\alpha^{\prime}\left(k_{x} k_{x}^{\prime}+k_{y} k_{y}^{\prime}\right), \\
\alpha=t(t+2 \gamma), \quad \alpha^{\prime}=\gamma(1-t) .
\end{gathered}
$$

We notice that the bare mass of the theory is given by

$$
m_{\mathrm{BC}}=1-2 t-t^{2}+g_{0}=m_{\text {Ising }}+g_{0},
$$

where $m_{\text {Ising }}=1-2 t-t^{2}$ is the Ising mass which vanishes at the critical value $\tanh \left(K_{\mathrm{c}}\right)=\sqrt{2}-1$ corresponding to the second order transition point $T_{\mathrm{c}}=2 / \ln (1+\sqrt{2}) \simeq 2.2691851$ in units of $J / k_{\mathrm{B}}$. In the $\mathrm{BC}$ model, the critical temperature is shifted by the parameter $g_{0}$ which depends on 
the temperature and $\Delta_{0}$. The location of the second order critical line goes from the previous Ising critical value $T_{\mathrm{c}}=2 / \log (1+\sqrt{2})$ when $\Delta_{0} \rightarrow-\infty$ to the zero temperature point $\left(T_{\mathrm{c}}=0, \Delta_{0}=2\right)$ continuously where the transition can be proved to be first order by a simple energetic argument. In figure 1 , we have reported the critical line given by $m_{\mathrm{BC}}=0$ and the different numerical results found in literature [12]. In general, the agreement is good, which validates the fermionic theory giving a bare mass vanishing at locations close to critical point values found by numerical methods. The presence of a tricritical point is induced by the interaction term (34) which renders the second order line instable. To see why, let us consider the infrared limit on the critical line. The spectrum is given by the lowest terms of an expansion of the effective action with respect to kinetic terms, an the contribution to the partition function, in the Fourier space, is the product of partial integrals $Z_{\mathbf{k}}$ such as $Z=\prod_{\mathbf{k}} Z_{\mathbf{k}}$, up to the second order in the momentum $\mathbf{k}$. For the Ising model and for small momenta, the factors $Z_{\mathbf{k}}$ are exactly of the form $\left(m_{\text {Ising }}+A k^{2}\right)$, with $A$ a constant equal to $t\left(1-t^{2}\right)$ [18]. The coefficient in front of the term $\mathbf{k}^{2}$ in factors $Z_{\mathbf{k}}$ can by described physically as a stiffness coefficient. For the Ising model, the stiffness is always strictly positive even at the critical point. In the $\mathrm{BC}$ case, however, we have a line of critical points as $\Delta_{0}$ varies from negative to positive values up to $\Delta_{0}=2$. The effect of the interaction potential (34) is to modify the expression of the stiffness, which now is no more constant but depends on the angle of the vector $\mathbf{k}$ as well as the temperature and $\Delta_{0}$ (see reference [12] for explicit details). The result is that in the $\mathrm{BC}$ case the effective stiffness coefficient vanishes at some point on the critical line, at a value close to $\Delta_{0}=2$, which eventually indicates the presence of a tricritical point. It can be shown that the partition function can be indeed written as a product over the Fourier modes $Z=\prod_{\mathbf{k} \in S} Z_{\mathbf{k}}$ with

$$
Z_{\mathbf{k}}=m_{\mathrm{BC}}^{2}+k^{2}\left[A+B \sin 2 \theta_{k}\right],
$$

$\theta_{k}$ being the angle of the vector $\mathbf{k}$, and $A$ and $B$ depending on temperature and $\Delta_{0}$. As long as $|A|$ is larger than $|B|$ on the critical line, the transition is second order. A singular point can be reached if $A^{2}=B^{2}$, in such case $Z_{\mathbf{k}}$ are not all strictly positive if $m_{\mathrm{BC}}=0$. Beyond this point the effective action (30) is not sufficient to describe the critical properties of the model. If we compare the fermionic description of the BC model to a bosonic Ginsburg-Landau $\Phi^{6}$ theory describing the first order transitions, the presence of a tricritical point would be equivalent to the fact that both coefficients of $\Phi^{2}$ and $\Phi^{4}$ terms vanish.

\subsection{Critical behavior of the $B C$ model: diagrammatic expansion}

In this section, we further analyze the effect of the interaction potential $V_{\mathbf{k}, \mathbf{k}^{\prime}}$ on the renormalized mass, in particular the shift of the critical temperature which was in reference [12] assumed to be given by the point where the bare mass $m_{\mathrm{BC}}$ vanishes. We would like, in particular, to apply diagrammatic expansion of the effective action (30). To this end, it is useful to express the Ising part of the action in terms of Nambu-Gorkov representation of the fermions [23,24], using the two-component objects

$$
\Phi_{\mathbf{k}}=\left(\begin{array}{c}
c_{\mathbf{k}} \\
\bar{c}_{-\mathbf{k}}
\end{array}\right), \quad \bar{\Phi}_{\mathbf{k}}=\left(\bar{c}_{\mathbf{k}}, c_{-\mathbf{k}}\right) .
$$

Formally, the Green functions can be defined within this representation by $2 \times 2$ matrices

$$
\hat{G}(\mathbf{k})=\left\langle\Phi_{\mathbf{k}} \bar{\Phi}_{\mathbf{k}} \tau_{3}\right\rangle
$$

where $\tau_{3}$ is the Pauli matrix

$$
\tau_{3}=\left(\begin{array}{cc}
1 & 0 \\
0 & -1
\end{array}\right)
$$

The unperturbed part of the Green function $\hat{G}_{0}$ is evaluated using the elements of the non-diagonal but quadratic Ising action (32):

$$
\hat{G}_{0}(\mathbf{k})=\frac{-1}{\left|m_{k}\right|^{2}+4 t^{2} k_{x} k_{y}}\left(\begin{array}{cc}
\bar{m}_{k} & 2 \mathrm{i} t k_{y} \\
2 \mathrm{i} t k_{x} & m_{k}
\end{array}\right),
$$


where the momentum-dependent mass is defined by $m_{k}=m_{\mathrm{BC}}+\mathrm{i} t(t+1)\left(k_{x}-k_{y}\right)$. The inverse is given by

$$
\hat{G}_{0}^{-1}(\mathbf{k})=\left(\begin{array}{cc}
-m_{k} & 2 \mathrm{i} t k_{y} \\
2 \mathrm{i} t k_{x} & -\bar{m}_{k}
\end{array}\right)
$$

With this representation and the unperturbed Green function, we can write the Ising part as

$$
S_{\text {Ising }}=\sum_{k \in S} \bar{\Phi}_{\mathbf{k}} \tau_{3} \hat{G}_{0}^{-1}(\mathbf{k}) \Phi_{\mathbf{k}}
$$

where the set $S$ contains half the momenta of the Brillouin zone. It is defined by the rule that if $\mathbf{k} \in S$, then $\mathbf{- k}$ does not belong to $S$. The interaction part can be put, after some algebra, into the following form

$$
S_{\mathrm{int}}=-g_{0} \frac{1}{4 L^{2}} \sum_{\mathbf{k}^{\prime}, \mathbf{k}^{\prime \prime}, \mathbf{q}}\left(\bar{\Phi}_{\mathbf{k} "-\mathbf{q}} \tau_{3} \hat{V}_{\mathbf{k}^{\prime \prime}-\mathbf{q}, \mathbf{k}} " \Phi_{\mathbf{k}} "\right)\left(\bar{\Phi}_{\mathbf{k}^{\prime}+\mathbf{q}} \tau_{3} \Phi_{\mathbf{k}^{\prime}}\right)
$$

where the sum is not restricted to the ensemble $S$. We define the potential matrix $\hat{V}$ by

$$
\hat{V}_{\mathbf{k} "-\mathbf{q}, \mathbf{k} "}=\left(\begin{array}{cc}
V_{\mathbf{k}}, \mathbf{k} "-\mathbf{q} & 0 \\
0 & V_{\mathbf{k} "-\mathbf{q}, \mathbf{k} "}
\end{array}\right) .
$$

The two diagonal elements of this matrix are not equal since $V_{\mathbf{k}, \mathbf{k}^{\prime}}$ is not symmetric by exchange of the two momenta $\mathbf{k}$ and $\mathbf{k}^{\prime}$ except when $\mathbf{k}=\mathbf{k}^{\prime}$.

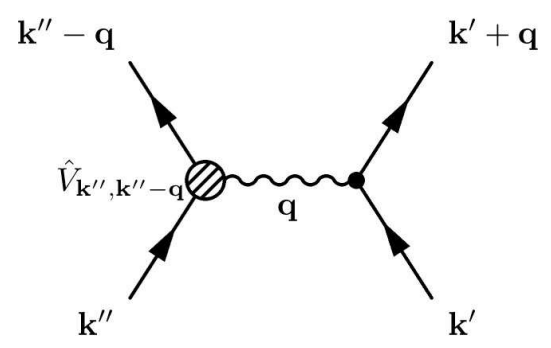

Figure 2. Representation of the interaction part of the potential. The blob represents the potential interaction with incoming vector $\mathbf{k}^{\prime \prime}$ and outgoing $\mathbf{k}^{\prime \prime}-\mathbf{q}$.

We now perform a diagrammatic expansion with respect to $g_{0}$ of the perturbed Green function $\hat{G}(\mathbf{k})$ which will allow us to compute the corrections to the mass, i. e. the shift of the critical temperature, by identification of the diagonal elements of the inverse propagator $\hat{\Gamma}(\mathbf{k})=\hat{G}(\mathbf{k})^{-1}$. The graphical representation of the matrix potential in terms of diagram is displayed in figure 2 . To do so we formally follow the Feynman rules which lead to the Dyson equation of the inversepropagator in terms of the self-energy $\hat{\Sigma}(\mathbf{k})$ :

$$
\hat{\Gamma}(\mathbf{k})=\hat{G}(\mathbf{k})^{-1}=\hat{G}_{0}(\mathbf{k})^{-1}-\hat{\Sigma}(\mathbf{k}) .
$$

The first terms contributing to the self-energy are given in figure 3 :

$$
\begin{aligned}
\hat{\Sigma}(\mathbf{k})= & -g_{0} \frac{1}{4 L^{2}} \sum_{\mathbf{q}}\left[\hat{V}_{\mathbf{k}, \mathbf{k}+\mathbf{q}} \hat{G}_{0}(\mathbf{k}+\mathbf{q})+\hat{G}_{0}(\mathbf{k}-\mathbf{q}) \hat{V}_{\mathbf{k}-\mathbf{q}, \mathbf{k}}\right] \\
& -g_{0} \frac{1}{4 L^{2}} \sum_{\mathbf{q}}\left[\hat{V}_{\mathbf{k}, \mathbf{k}} \operatorname{Tr} \hat{G}_{0}(\mathbf{q})+\hat{V}_{\mathbf{q}, \mathbf{q}} \operatorname{Tr} \hat{G}_{0}(\mathbf{q})\right] .
\end{aligned}
$$

The renormalized mass $m_{R}$ is given in the limit when $k$ is zero by the diagonal components of the inverse-propagator $\Gamma_{11}(0)=\Gamma_{22}(0)$. In this limit, only one diagram is not vanishing, which 

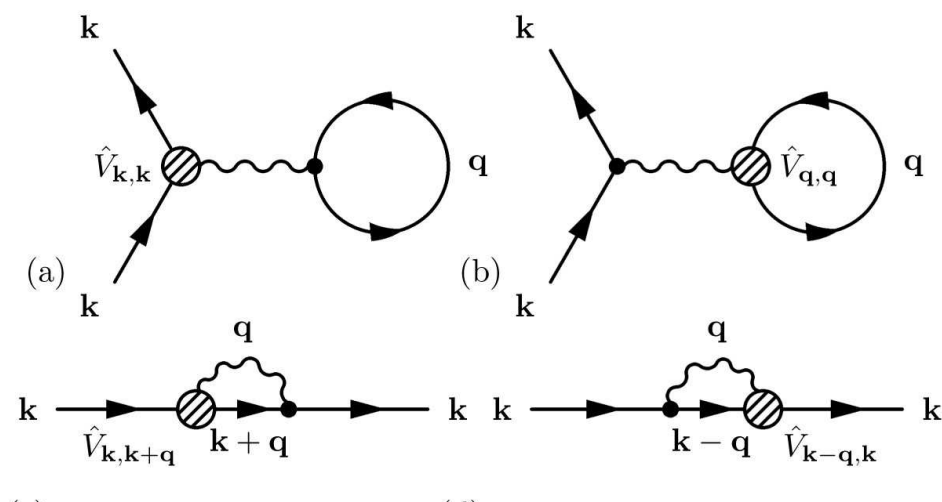

(c)

(d)

Figure 3. The four diagrams appearing at the lowest order in $g_{0}$. Only diagram (b) contributes to the mass in the low momentum limit $k \rightarrow 0$.

corresponds to the diagram (b) of figure 3 :

$$
\begin{aligned}
m_{R} & =\Gamma_{11}(0)=m_{\mathrm{BC}}+g_{0} \frac{1}{4 L^{2}} \sum_{\mathbf{q}} V_{\mathbf{q}, \mathbf{q}} \operatorname{Tr} \hat{G}_{0}(\mathbf{q}) \\
& =m_{\mathrm{BC}}-m_{\mathrm{BC}} \times g_{0} \frac{1}{2 L^{2}} \sum_{\mathbf{q}} \frac{V_{\mathbf{q}, \mathbf{q}}}{m_{\mathrm{BC}}^{2}+t^{2}(1+t)^{2}\left(q_{x}-q_{y}\right)^{2}+4 t^{2} q_{x} q_{y}} .
\end{aligned}
$$

The last sum over $q$ can be evaluated in the continuous limit $L \rightarrow \infty$. Setting $\mathbf{q}=2 \pi\left(\frac{m}{L}, \frac{n}{L}\right)$, we define for large $L$ the two following integrals

$$
\begin{aligned}
& I_{1}\left(m_{\mathrm{BC}}\right)=\frac{g_{0}}{8 \pi^{2}} \int_{0}^{2 \pi} \int_{0}^{2 \pi} \mathrm{d} q_{x} \mathrm{~d} q_{y} \frac{q_{x} q_{y}}{m_{\mathrm{BC}}^{2}+t^{2}(1+t)^{2}\left(q_{x}-q_{y}\right)^{2}+4 t^{2} q_{x} q_{y}} \\
& I_{2}\left(m_{\mathrm{BC}}\right)=\frac{g_{0}}{8 \pi^{2}} \int_{0}^{2 \pi} \int_{0}^{2 \pi} \mathrm{d} q_{x} \mathrm{~d} q_{y} \frac{q_{x}^{2}+q_{y}^{2}}{m_{\mathrm{BC}}^{2}+t^{2}(1+t)^{2}\left(q_{x}-q_{y}\right)^{2}+4 t^{2} q_{x} q_{y}}
\end{aligned}
$$

These two quantities are finite when $m_{\mathrm{BC}}$ vanishes. To see why, we can consider polar coordinates $q_{x}=q \cos \theta$ and $q_{y}=q \sin \theta$, so that, near the origin $q=0$ the second integral for example behaves like

$$
I_{2}\left(m_{\mathrm{BC}}\right) \propto \frac{g_{0}}{8 \pi^{2} t^{2}(1+t)^{2}} \int_{0} \int_{0}^{2 \pi} q \mathrm{~d} q \mathrm{~d} \theta \frac{1}{\left\{m_{\mathrm{BC}} / q t(1+t)\right\}^{2}+1+\left\{-1+2 /(1+t)^{2}\right\} \sin (2 \theta)} .
$$

When $m_{\mathrm{BC}}=0$, this integral is finite since there is no singularity in the denominator. Indeed when $\theta= \pm \pi / 4$ the modulus of the term $-1+2 /(1+t)^{2}$ is strictly less than one on the critical line. This would not be the case if the last term $4 t^{2} q_{x} q_{y}$ in the denominator and coming from the off-diagonal part of the Green function was absent. In this case the integrals would be singular in the limit of small mass $m_{\mathrm{BC}}$, the denominator would instead be equal to $\left\{m_{\mathrm{BC}} / q t(1+t)\right\}^{2}+1-\sin (2 \theta)$, and the singular part would behave like $1 /\left|m_{\mathrm{BC}}\right|$ by a simple scaling argument, which would cancel the other mass term $m_{\mathrm{BC}}$ in front of the integrals (47). Then the renormalized mass would be shifted by a finite quantity, as well as the critical line. Here the renormalization only concerns the total coefficient of $m_{\mathrm{BC}}$ and this does not affect the critical line location:

$$
m_{R} \simeq m_{\mathrm{BC}}\left(1-\alpha I_{1}(0)+\alpha^{\prime} I_{2}(0)\right)
$$

A plot of the positive ratio $m_{R} / m_{\mathrm{BC}}$ evaluated at criticality $m_{\mathrm{BC}}=0$ as function of $\Delta_{0}$ is given in figure 4. It is close to unity for almost all values of $\Delta_{0}$. 


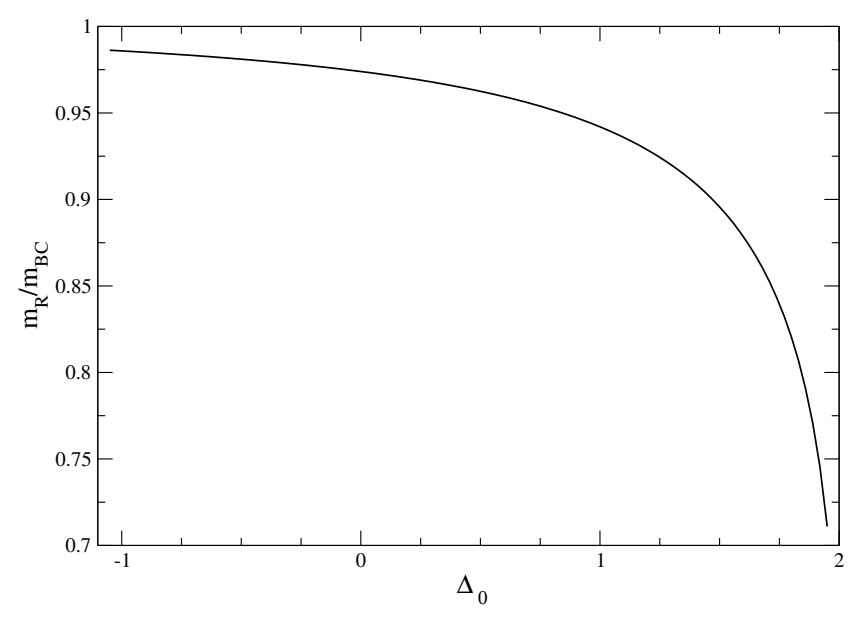

Figure 4. Plot of the coefficient renormalizing the BC bare mass as function of $\Delta_{0}$ on the critical line. The values are close to unity except near the region of the terminating point $\Delta_{0}=2$ where $g_{0}$ is not small.

This analysis shows that corrections to critical temperatures are indeed small and are of the order higher than $g_{0}$. A further analysis should be carried out to obtain a finite shift by considering more complex diagrams. It is also supported by analytical values of $m_{\mathrm{BC}}=0$ which are close to numerical results reported in figure 1.

\section{Extension to other spin-S models: generalization of the bare mass}

The previous bare mass computed in the $\mathrm{BC}$ model, $m_{\mathrm{BC}}$, allowed us to obtain a precise description of the second-order critical line in the phase diagram. This was obtained by taking the limit of low momentum in the effective action, see (30). This can be generalized for any value of the spin $S$, in particular for higher values of $S$. The equations obtained in section 2, equations (16) and (17), are general for they represent the fermionization of any spins-S model.

The construction of the fermionic action is, however, not an easy task, unlike the BC model which is a simpler case, but we expect to be able to extract a bare mass associated to non-kinetic terms, or term involving derivatives with respect to space variables. At first approximation, we assume that the partition function and the free energy are singular in the low momentum limit when this bare mass vanishes. In the continuum limit, the $c$ 's coefficients defined by relations (13) can be rewritten using formal derivatives, such as $c_{m n}^{1}=x_{q}\left(\bar{a}_{m n}^{q}-\partial_{x} \bar{a}_{m n}^{q}\right)$, etc. The derivatives contribute only to the kinetic energy and not to the bare mass. Keeping the first terms of the expansion, $c_{m n}^{1} \simeq x_{q} \bar{a}_{m n}^{q}$ (as well as for the other coefficients $c$ 's), the weights $W_{m n}$ become uncoupled in the sense they contain variables depending only on local site $(m, n)$ and we define the mass $m_{S}$ as:

$$
m_{\mathrm{S}} \equiv u_{0}^{2} \int\left[\prod_{\alpha=1}^{q} \mathrm{~d} \bar{a}_{m n}^{\alpha} \mathrm{d} a_{m n}^{\alpha} \mathrm{d} \bar{b}_{m n}^{\alpha} \mathrm{d} b_{m n}^{\alpha} \mathrm{e}^{a_{m n}^{\alpha} \bar{a}_{m n}^{\alpha}+b_{m n}^{\alpha} \bar{b}_{m n}^{\alpha}}\right] W_{m n}
$$

with $c_{m n}^{1} \simeq x_{q} \bar{a}_{m n}^{q}, \cdots, c_{m n}^{q} \simeq x_{1} \bar{a}_{m n}^{1}$ and $c_{m n}^{q+1} \simeq x_{q} \bar{b}_{m n}^{q}, \cdots, c_{m n}^{2 q} \simeq x_{1} \bar{b}_{m n}^{1}$. The integral can be evaluated exactly by noticing for example that the arguments of the exponential $b_{m n}^{\alpha} \bar{b}_{m n}^{\alpha}$ can be combined with a $a_{m n}^{\alpha}\left(x_{\alpha} \bar{a}_{m n}^{\alpha}\right)$ that appears in some of the $q_{k=1 \ldots 2 q}^{(2 k)}$ products to give a contribution 
$x_{\alpha}$. Indeed using the Grassmann integration rules $\int \mathrm{d} a . a=1$ and $\int \mathrm{d} a .1=0$, we can write

$$
\begin{aligned}
\int \mathrm{d} \bar{a}_{m n}^{\alpha} \mathrm{d} & a_{m n}^{\alpha} \mathrm{d} \bar{b}_{m n}^{\alpha} \mathrm{d} b_{m n}^{\alpha} \mathrm{e}^{a_{m n}^{\alpha} \bar{a}_{m n}^{\alpha}+b_{m n}^{\alpha} \bar{b}_{m n}^{\alpha}} a_{m n}^{\alpha}\left(x_{\alpha} \bar{a}_{m n}^{\alpha}\right) \\
& =\int \mathrm{d} \bar{a}_{m n}^{\alpha} \mathrm{d} a_{m n}^{\alpha} \mathrm{d} \bar{b}_{m n}^{\alpha} \mathrm{d} b_{m n}^{\alpha}\left(1+a_{m n}^{\alpha} \bar{a}_{m n}^{\alpha}+b_{m n}^{\alpha} \bar{b}_{m n}^{\alpha}+a_{m n}^{\alpha} \bar{a}_{m n}^{\alpha} b_{m n}^{\alpha} \bar{b}_{m n}^{\alpha}\right) a_{m n}^{\alpha}\left(x_{\alpha} \bar{a}_{m n}^{\alpha}\right) \\
& =\int \mathrm{d} \bar{a}_{m n}^{\alpha} \mathrm{d} a_{m n}^{\alpha} \mathrm{d} \bar{b}_{m n}^{\alpha} \mathrm{d} b_{m n}^{\alpha} b_{m n}^{\alpha} \bar{b}_{m n}^{\alpha} a_{m n}^{\alpha}\left(x_{\alpha} \bar{a}_{m n}^{\alpha}\right)=x_{\alpha} .
\end{aligned}
$$

Also a term $a_{m n}^{\alpha} \bar{a}_{m n}^{\alpha}$ can be combined with $b_{m n}^{\alpha}\left(x_{\alpha} \bar{b}_{m n}^{\alpha}\right)$ to give the same contribution. Since the $q^{(2 k)}$ are ordered, there are also signs to take into account and coming from the fact the variables $c_{m n}^{\alpha}$ have to be moved in the correct order before integration. We obtain after some algebra the general relation

$$
m_{\mathrm{S}}=\sum_{k=0}^{2 q} \alpha_{k} R_{k}
$$

where we have defined the following quantities with initial condition $R_{0}=u_{0}^{2}$,

$$
R_{k}=\sum_{l=0}^{k} u_{l} u_{k-l} \sigma(l, k-l),
$$

and $\sigma(k, l)=1$ if $k$ and $l$ are both even, and $\sigma(k, l)=-1$ otherwise. We can apply this result to different cases to check the validity of this relation. For the Ising model $(S=1 / 2, q=1)$ $u_{0}=\operatorname{ch}(K / 4)$ and $u_{1}=4 \operatorname{sh}(K / 4)$, we obtain $m_{1 / 2}=2 \cosh (\Delta / 4)\left(u_{0}^{2}-u_{0} u_{1} / 2-u_{1}^{2} / 16\right)$, or

$$
m_{1 / 2}=2 \mathrm{e}^{\Delta / 4}[1-\operatorname{sh}(K / 2)],
$$

which vanishes at the Ising critical temperature $T_{\mathrm{c}} \simeq 0.567296$ or with the normalization $t_{\mathrm{c}} \equiv$ $T_{\mathrm{c}} / S^{2}=2.269185$, which is independent, as expected, of $\Delta_{0}$. For the Blume-Capel model $(S=$ $1, q=2)$ we have $m_{1}=1+2 \exp (\Delta)\left(1-2 u_{1}+2 u_{2}-u_{1}^{2}-2 u_{1} u_{2}+u_{2}^{2}\right)$, or more explicitly

$$
m_{1}=1+2 \mathrm{e}^{\Delta}[1-\operatorname{sh}(2 K)] .
$$

This mass is directly proportional to the mass $m_{\mathrm{BC}}$ found in the previous section. Indeed, we have the relation

$$
m_{\mathrm{BC}}=g_{0} m_{1}
$$

and, therefore, both masses vanish on the same line of critical points. The coefficient $g_{0}$ comes from a global rescaling of the Grassmann variables in the original weights $W_{m n}$ which leads to the coefficient $g_{0}^{-L^{2}}$ in the BC function partition (19) and (22), instead of the coefficient $\left(u_{0}\right)^{2 L^{2}}=1$ in front of (17). For $\Delta_{0}=0$ we find, in particular, that $t_{\mathrm{c}}=2 / \operatorname{arcsinh}(3 / 2) \simeq 1.673971$ (see table 1).

The other masses are deduced by iteration of formula (51) and (52). For higher values of spin $S$, we find:

$$
\begin{aligned}
m_{3 / 2} & =2 \mathrm{e}^{\Delta / 4}[1-\operatorname{sh}(K / 2)]+2 \mathrm{e}^{9 \Delta / 4}[1-\operatorname{sh}(9 K / 2)]+2 \mathrm{e}^{\Delta / 4}[1-\operatorname{sh}(K / 2)], \\
m_{2} & =1+2 \mathrm{e}^{\Delta}[1-\operatorname{sh}(2 K)]+2 \mathrm{e}^{4 \Delta}[1-\operatorname{sh}(8 K)], \\
m_{5 / 2} & =2 \mathrm{e}^{25 \Delta / 4}[1-\operatorname{sh}(25 K / 2)]+2 \mathrm{e}^{9 \Delta / 4}[1-\operatorname{sh}(9 K / 2)]+2 \mathrm{e}^{\Delta / 4}[1-\operatorname{sh}(K / 2)], \\
m_{3} & =1+2 \mathrm{e}^{9 \Delta}[1-\operatorname{sh}(18 K)]+2 \mathrm{e}^{4 \Delta}[1-\operatorname{sh}(8 K)]+2 \mathrm{e}^{\Delta}[1-\operatorname{sh}(2 K)] .
\end{aligned}
$$

For general spin $S$, we can extend the previous results to the formula

$$
m_{S}=\sum_{\sigma=-S}^{S} \mathrm{e}^{\Delta \sigma^{2}}\left[1-\operatorname{sh}\left(2 \sigma^{2} K\right)\right] .
$$


Table 1. Comparison of critical temperature values at $\Delta_{0}=0$, solutions of equation (57), with other methods found in bibliography.

\begin{tabular}{c|cccc}
\hline Spin S & $S=1 / 2$ & $S=1$ & $S=3 / 2$ & $S=2$ \\
\hline$q$ & 1 & 2 & 5 & 6 \\
$T_{\mathrm{c}}$ & 0.567296 & 1.673971 & 3.277561 & 5.351248 \\
$t_{\mathrm{c}}=T_{\mathrm{c}} / S^{2}$ & 2.269185 & 1.673971 & 1.456694 & 1.337812 \\
Refs. & $2.269[25]$ & $1.689[26], 1.695[19]$ & $1.461[27,25,28]$ & $1.336[25]$ \\
& & $1.694[25], 1.681[27]$ & & \\
\hline Spin S & $S=5 / 2$ & $S=3$ & $S=\infty$ & \\
\hline$q$ & 11 & 12 & $\infty$ & \\
$T_{\mathrm{c}}$ & 7.890888 & 10.894806 & $\infty$ & \\
$t_{\mathrm{c}}=T_{\mathrm{c}} / S^{2}$ & 1.262542 & 1.210534 & 0.925148 & \\
Refs. & $1.257[25]$ & $1.203[25]$ & $0.915[25,29]$ &
\end{tabular}

This is a simple result giving a precise location of the second-order critical lines by solving the equation of the bare mass $m_{S}=0$. For comparison we give tabulated values of $t_{\mathrm{c}}$ at $\Delta_{0}=0$ in table 1 for different values of $S$, and references to numerical results (Monte-Carlo simulations, high-temperature expansions) given in the literature. In general, the agreement is good up to $1 \%$ in most cases. For half-integer values of $S$, the models possess an asymptote in the $\left(T / S^{2}, \Delta_{0}\right)$ plane. Indeed, for $S=3 / 2$, for example, the equation given in (56) predicts the solution

$$
\Delta_{0}=-\frac{9 t}{8} \log \left[-\frac{1-\operatorname{sh}(2 / 9 t)}{1-\operatorname{sh}(2 / t)}\right]
$$

which is bounded by $t_{\mathrm{c}}=2 / 9 \log (1+\sqrt{2})=0.252131$ below which there is no continuation of the second-order critical line. In the limit of large integer $S$, the model defined in equation (1) is described by a continuous variable $-1<x_{m n}=S_{m n} / S<1$ and is called continuous Ising model. We can still obtain a finite value of the critical line by taking the asymptotic value of equation (57)

$$
m_{S \gg 1} \simeq S \sqrt{2 t} \int_{0}^{\sqrt{2 / t}} \mathrm{~d} x \mathrm{e}^{-\Delta_{0} x^{2} / 2}\left[1-\operatorname{sh}\left(x^{2}\right)\right],
$$

and, in particular, for $\Delta_{0}=0$, we have the following expansion for large $S$

$$
m_{S}\left(t, \Delta_{0}=0\right) \simeq a(t) S-1+\frac{4}{3 t S}+\frac{8}{21 t^{3} S^{6}}+\cdots
$$

with $a(t)=2-2 \sqrt{2 / t} \int_{0}^{\sqrt{2 / t}} \operatorname{sh}\left(x^{2}\right) \mathrm{d} x$. We observe that the rescaled mass $m_{S} / S$ vanishes in this case when $t_{\mathrm{c}}=0.925148$, in good agreement with numerical works for this model [25,29], and it is worth noting that equation (59) also possesses a non-trivial solution at $t=0$ which is simply given by $\Delta_{0}=4 / \sqrt{3}=2.309401$. This value is different from the value 2 expected for all finite $S$ models [8]. It can be suggested that there also exists a tricritical point before this non-physical value is reached.

\section{Conclusion}

In this review paper, we have presented a method which tries to operate a correspondence between classical spin models and fermionic systems. We have extended Plechko's method $[6,10]$ based on Ising model to generalized spin-S systems. The method is based on the projection onto $q$ polynomial components, $q$ depending specifically on the value of spin $S$, of Boltzmann local weights given by equation (2). Then the introduction of $2 q$ pairs of Grassmann variables per site and the use of special symmetries such as mirror and associative symmetries in 2D for Grassmannian 
objects allows us to perform exactly the sum over the spin variables. This gives a representation of spin-S models in terms of fermionic multiple integrals (17). Effective actions can in principle be deduced from this representation. We have shown that such an action can be built exactly for the Blume Capel model $S=1$ (30) and the bare mass (35) gives accurate description of the second-order critical line. We have seen that there is no shift of this mass due to the effect of quartic potential of the effective theory at the lowest order expansion in the coupling parameter $g_{0}$, implying that corrections to the critical temperature may be indeed small. This quartic potential is, however, responsible for the presence of a tricritical point, rendering the second order line instable by changing the sign of the stiffness coefficient or making the free-fermion spectrum itself instable. For general spin-S model, the bare mass can also be generalized and calculated directly in the low momentum limit (51) without knowing the full effective action, and it still gives an accurate description of second-order critical points even in the limit of the continuous Ising model.

\section{References}

1. Lieb E.H., Schultz T.D., Mattis D.C., Ann. Phys, 1961,16, 407.

2. Schultz T.D., Mattis D.C., Lieb E.H., Rev. Mod. Phys., 1964,36, 856.

3. Jordan P., Wigner E., Z. Phys., 1928 47, 631.

4. Fisher M.E., J. Math. Phys., 1966, 7, 1776.

5. Bugrij A.I. Electron-Electron Correlation Effects in Low-Dimentional Conductors and Superconductors. Editors: Ovchinnikov A.A., Ukrainskii I.I. Heidelberg, Springer-Verlag, 1991, p. 135-151.

6. Plechko V.N., Theoretical and Mathematical Physics, 1985, 64, 748-756.

7. Nojima K., Int. J. Mod. Phys. B, 1998, 12, 1995.

8. Fortin J.-Y., Clusel M., Phys. Rev. B, 2008, 78, 172402.

9. Blume M., Emery V.J., Griffiths R.B., Phys. Rev. A, 1971, 4, 1071.

10. Plechko V.N., Sov. Phys. Doklady, 1985, 30, 271.

11. Berezin F.A. The Method of Second Quantization. Academic Press, 1966.

12. Clusel M., Fortin J.-Y., Plechko V.N., J. Phys. A: Math. Theor., 2008, 41, 405004.

13. Liaw T.M., Huang M.C., Lin S.C., Wu M.C., Phys. Rev. B, 1999, 60, 12994.

14. Wu M.C., Hu C.K., J. Phys. A: Math. Gen., 2002, 35, 5189.

15. Barbaro M.B., Molinari A., Palumbo F., Nucl. Phys. B, 1997, 487, 492.

16. Plechko V.N. Anticommuting integrals and fermionic field theories for two-dimensional Ising models. A talk given at the VII International Conference on Symmetry Methods in Physics, ICSMP-95, Dubna, July 10-16, 1995. See also hep-th/9607053.

17. Plechko V.N., Phys. Lett. A, 1998, 239, 289.

18. Plechko V.N., J. Phys. Studies, 1999, 3, 312.

19. da Silva R., Roberto, Alves N.A., Drugowich de Felício J.R., Phys. Rev. E, 2002, 66, 026130.

20. Silva C.J., Caparica A.A., Plascak J.A., Phys. Rev. E, 2006, 73, 036702.

21. Xavier J.C., Alcaraz F.C., Pena Lara D., Plascak J.A., Phys. Rev. B, 1998, 57, 11575.

22. Beale P.D., Phys. Rev. B, 1986, 33, 1717.

23. Mattuck Richard D. A Guide to Feynman Diagrams in the Many-Body Problem. Courier Dover Publications, 1992.

24. Tsvelik A.M. Quantum Field Theory in Condensed Matter Physics. Cambridge, 1995.

25. Butera P., Comi M., Guttmann A.J., Phys. Rev. B, 2003, 67, 054402.

26. Fox P.F., Guttmann A.J., J. Phys. C, 1973, 6, 913.

27. Xavier J.C., Alcaraz F.C., Lara D.P., Plascak J.A., Phys. Rev. B, 1998, 57, 11575.

28. Grandi B.C.S., Figueiredo W., Phys. Rev. E, 2004, 70, 056109.

29. Bialas P., Blanchard P., Fortunato S., Gandolfo, D., Satz H., Nucl. Phys. B, 2000, 583, 368. 


\title{
Грасманові техніки, застосовані до класичних спінових систем
}

\author{
М. Клюсел ${ }^{1}$, Ж.-І. Фортен ${ }^{2}$
}

1 Відділ фізики і Центр досліджень м'якої речовини, Університет Нью-Йорка, 4 площа Вашінгтона, Нью-Йорк NY 10003, США

2 Інститут Жана Лямура, відділ фізики матерії і матеріалів, група статистичної фізики CNRS,

Університет Нансі, поштова скринька 70239, F-54506 Вандевр лє Нансі Седекс, Франція

Отримано 22 квітня 2009 р., в остаточному вигляді - 20 травня 2009 р.

Ми оглядаємо задачі, у яких використовуються грасманові техніки для класичних спінових систем у двох вимірах. Ці техніки є корисними для встановлення точної відповідності між класичними спіновими гамільтоніанами і теоретико-польовими ферміонними діями. Це дає змогу краще зрозуміти критичну поведінку цих моделей у термінах неквадратичних ефективних дій, на які можна дивитися, як на розширення вільноферміонної моделі Ізинга. У межах цього методу ідентифікація затравочної маси дозволяє виконати точну оцінку критичних точок чи ліній, що підтверджується результатами Монте Карло чи діаграмних технік.

Ключові слова: грасманова алгебра, спінові системи, критичні явища

PACS: 02.30.Ik, 05.50.+q, 05.70.Fh 